\title{
Vitamina A em dieta de juvenis de pacu cultivados em tanques-rede
}

\author{
Arcangelo Augusto Signor ${ }^{(1)}$, Flavia Renata Potrich ${ }^{(2)}$, Aldi Feiden ${ }^{(3)}$, \\ Wilson Rogério Boscolo(3), Ricardo Pereira Ribeiro(4) e Lauro Vargas ${ }^{(4)}$
}

\begin{abstract}
(1)Instituto Federal do Paraná, Avenida Araucária, o 780, CEP 85870-000 Foz do Iguaçu, PR. E-mail: arcangelo.signor@ifpr.edu.br (2)Universidade Estadual do Oeste do Paraná (Unioeste), Rua Pernambuco, no 1.777, CEP 85960-000 Marechal Cândido Rondon, PR. E-mail: flaviarenatapotrich@gmail.com ${ }^{(3)}$ Unioeste, Rua da Faculdade, № 645, CEP 85903-000 Toledo, PR. E-mail: aldifeiden@gmail.com, wrboscolo@bol.com.br (4)Universidade Estadual de Maringá, Avenida Colombo, no 5.790, CEP 87020-900 Maringá, PR. E-mail: rpribeiro@uem.br, Ivargas@uem.br
\end{abstract}

Resumo - O objetivo deste trabalho foi avaliar o efeito da suplementação de vitamina A na alimentação de juvenis de pacu cultivados em tanques-rede. Foram utilizados 2.000 peixes, com peso inicial médio de $66,93 \pm 15,03 \mathrm{~g}$, distribuídos em delineamento inteiramente casualizado, em 20 tanques-rede de $5,0 \mathrm{~m}^{3}$, com cinco tratamentos e quatro repetições. As rações foram formuladas com 0, 3.000, 6.000, 9.000 e 12.000 UI de vitamina A por quilograma de dieta, por meio da suplementação com acetato de retinol. $\mathrm{O}$ arraçoamento foi realizado às 8:30, 13:30 e 17:30 h, até a saciedade aparente dos animais. Não foram observadas diferenças quanto aos parâmetros de desempenho produtivo, rendimento e composição química da carcaça, e aos parâmetros hematológicos e lipídios no fígado dos peixes que foram alimentados com diferentes níveis de vitamina A. A suplementação de vitamina A em dietas para juvenis de pacu criados em tanques-rede não influencia o desempenho produtivo dos peixes.

Termos para indexação: Piaractus mesopotamicus, exigência vitamínica, peixes nativos, vitaminas lipossolúveis.

\section{Vitamin A in a diet of juvenile pacu reared in cages}

\begin{abstract}
The objective of this work was to evaluate the effect of vitamin A supplementation in a diet of juvenile pacu reared in cages. Two thousand fish were used, with $66.93 \pm 15.03 \mathrm{~g}$ initial weight, and distributed in a completely randomized design in 20 cages of $5.0 \mathrm{~m}^{3}$, with five treatments and four replicates. The diets were formulated with $0,3,000,6,000,9,000$ and 12,000 UI of vitamin A per kilogram of diet, supplemented by retinyl acetate. Feeding was carried at 8:30; 1:30 and 5:30 h, until apparent satiety of animals. No difference was observed for the parameters of production performance, yield and chemical composition of the carcass, and for the blood and lipids parameters in the liver of fish fed different levels of vitamin A. The vitamin A supplementation in diets for juvenile pacu reared in cages has no effect on fish growth performance.
\end{abstract}

Index terms: Piaractus mesopotamicus, vitamin requirement, native fish, fat-soluble vitamins.

\section{Introdução}

O pacu (Piaractus mesopotamicus), peixe endêmico das bacias dos Rios Paraná e Paraguai é uma importante espécie comercializada na região (Jomori et al., 2003). Entretanto, vem sofrendo redução progressiva do estoque populacional em seu habitat (Lopera Barrero et al., 2008). Porém, o interesse em sistemas de cultivo nas regiões Oeste e Sudoeste do Paraná, tanto para a pesca esportiva quanto para a produção de carne cresce de forma significativa em razão do elevado valor comercial, rusticidade ao manejo, precocidade, rápido crescimento e adaptação aos sistemas produtivos desta espécie (Abimorad \& Carneiro, 2004; Furuya et al., 2008). O pacu altera sua alimentação de acordo com a sazonalidade da disponibilidade de alimentos e, em sistemas de cultivo, são alimentados com rações comerciais (Rodrigues et al., 2010).

A vitamina A participa de inúmeras funções nos organismos e está envolvida na síntese de algumas glicoproteínas e glicosaminoglicanos, que agem como hormônios esteroides na regulação do crescimento e diferenciação celular. Importante para a visão, reprodução, desenvolvimento embrionário e por atuação na resposta imune, a vitamina A influencia o crescimento e as deformações ósseas dos peixes (Lall \& Lewis-McCrea, 2007; Fernandez et al., 2009). A vitamina A é absorvida ao longo do intestino delgado com os lipídios provenientes das dietas; assim, condições favoráveis para a absorção de gorduras 
proporcionam o aumento da absorção destas vitaminas (Nutrient requirements of warmwater fishes and shellfishes, 1993).

Embora, tenham sido elencadas inúmeras atribuições positivas da vitamina $A$, a pesquisa sobre seu uso na alimentação para peixes, no Brasil, é escassa, da qual se podem destacar os trabalhos sobre tilápia-do-nilo (Oreochromis niloticus), de Campeche et al. (2009) e Guimarães (2009), que recomendam 4.800 e 4.704 UI de vitamina A por quilograma de ração, respectivamente, e o trabalho sobre larvas de jundiá (Rhamdia quelen), de Peil et al. (2007), que recomendam de 3.000 a 5.300 UI de vitamina A por quilograma de ração, para um bom desempenho produtivo dos peixes. Assim, avaliar o nível ideal de vitamina A para o pacu, visa complementar as informações e contribuir para o desenvolvimento de rações que atendam à exigênica da espécie.

O objetivo deste trabalho foi avaliar o efeito da suplementação de vitamina A na dieta de juvenis de pacu sobre o desempenho zootécnico e sobre as características de carcaça, deposição no fígado e parâmetros sanguíneos de pacu, cultivado em tanques-rede.

\section{Material e Métodos}

O experimento foi realizado no Centro de Desenvolvimento de Tecnologia para Piscicultura em Tanques-rede, localizado no Refúgio Biológico do Município de Santa Helena, PR, entre 245' $45^{\prime \prime}$, $54^{\circ} 24^{\prime} 16^{\prime \prime} \mathrm{W}, 24^{\circ} 54^{\prime} 12^{\prime \prime} \mathrm{S}, 5^{\circ} 22^{\prime} 18^{\prime \prime} \mathrm{W}$, e $24^{\circ} 53^{\prime} 23^{\prime \prime} \mathrm{S}$, $54^{\circ} 23^{\prime} 44^{\prime \prime} \mathrm{W}$, durante 90 dias entre os meses de março a junho.

Para a execução do experimento, o suplemento mineral e vitamínico foi formulado com 0, 3.000, $6.000,9.000$ e 12.000 UI de vitamina A, por quilograma da dieta, com acetato de retinol Microvit A Supra 1000 (Adisseo Brasil Nutrição Animal Ltda., Santo André, SP, Brasil) (Tabela 1).

Foram utilizados 2.000 juvenis de pacu, com $66,93 \pm 15,03$ g de massa e 14,59 $\pm 1,07 \mathrm{~cm}$ de comprimento, distribuídos em delineamento inteiramente casualizado, em 20 tanques-rede de $5,0 \mathrm{~m}^{3}$, com cinco tratamentos (níveis de vitamina A) e quatro repetições. A unidade experimental considerada foi o tanque com 100 peixes ( 20 peixes $\mathrm{m}^{-3}$ ).

A ração foi formulada com $26 \%$ de proteína bruta (PB) e $3.000 \mathrm{kcal} \mathrm{kg}^{-1}$ de energia digestível (ED). Para a confecção das rações, os alimentos (milho, farelo de soja, farelos de trigo, farinha de vísceras de aves e farinha de peixe) foram pesados, acrescentados no misturador vertical e moídos em moinho tipo martelo até atingir diâmetro de $0,8 \mathrm{~mm}$. Posteriormente, em misturador horizontal, foram homogeneizados e adicionados micronutrientes, suplemento mineral e vitamínico, antifúngico e, por último, o óleo. As rações foram submetidas ao processo de extrusão, para obtenção de péletes de $4,0 \mathrm{~mm}$, em seguida foram dispostas em um secador horizontal, embaladas e identificadas. $\mathrm{O}$ arraçoamento foi realizado três vezes ao dia, às 8:30, 13:30 e 17:30 h, até a saciedade aparente dos animais. O consumo alimentar dos peixes foi acompanhado por meio de pesagem e reposição diária das rações fornecidas aos peixes.

Os parâmetros de qualidade de água foram medidos quinzenalmente, e a temperatura $\left({ }^{\circ} \mathrm{C}\right)$ e a transparência da água $(\mathrm{m})$ foram monitoradas diariamente pela manhã $(8: 30 \mathrm{~h})$ e à tarde $(17: 30 \mathrm{~h})$. Os parâmetros de temperatura $\left(23,07 \pm 3,18^{\circ} \mathrm{C}\right)$, $\mathrm{pH}(7,72 \pm 0,23)$, transparência $(1,56 \pm 0,43 \mathrm{~m})$, oxigênio dissolvido $\left(6,85 \pm 0,26 \mathrm{mg} \mathrm{L}^{-1}\right)$ e condutividade elétrica

Tabela 1. Composição percentual e química da ração-base.

\begin{tabular}{lc}
\hline Ingrediente & Composição (\%) \\
\hline Milho em grão & 36,821 \\
Farelo de trigo & 20,000 \\
Farelo de soja & 20,000 \\
Farinha de vísceras de aves & 13,000 \\
Farinha de peixe & 7,296 \\
Calcário calcítico & 0,976 \\
Óleo de soja & 0,696 \\
Suplemento mineral e vitamínico(1) & 0,500 \\
Sal comum & 0,300 \\
DL-metionina & 0,205 \\
Antifúngico (propionato de cálcio) & 0,100 \\
L-lisina-HCL & 0,086 \\
Antioxidante (BHT) & 0,020 \\
\hline Total & 100 \\
\hline Composição química & 88,0 \\
Matéria seca & 26,0 \\
Proteína bruta & 3,90 \\
Gordura & 7,30 \\
Matéria mineral & \\
\hline
\end{tabular}

(1) Níveis de garantia por quilograma do produto: vitamina D3, $400.000 \mathrm{UI}$; vitamina E, $30.000 \mathrm{UI}$; vitamina $\mathrm{K} 3,2.000 \mathrm{mg}$; vitamina B1, $4.000 \mathrm{mg}$; vitamina B2, $4.000 \mathrm{mg}$; vitamina B6, $2.000 \mathrm{mg}$; vitamina B12, $8 \mathrm{mg}$; ácido fólico, $1.000 \mathrm{mg}$; pantotenato de cálcio, $1.000 \mathrm{mg}$; vitamina C, $60.000 \mathrm{mg}$; biotina, $200 \mathrm{mg}$; inositol, 20.000; niacina, 20.000; colina, $100.000 \mathrm{mg}$; Co, $140 \mathrm{mg}$; Cu, $2.000 \mathrm{mg}$; Fe, $16.000 \mathrm{mg}$; I, $200 \mathrm{mg}$; Mn, $10.000 \mathrm{mg}$; Se, $80 \mathrm{mg} ; \mathrm{Zn}, 16.000 \mathrm{mg}$. 
$\left(40,52 \pm 1,31 \mu \mathrm{S} \mathrm{cm}^{-1}\right)$ permaneceram dentro de valores aceitáveis para o desenvolvimento dos peixes (Boyd, 1990).

Aos 90 dias do experimento, realizou-se a coleta de sangue para análises hematológicas; para tanto, oito peixes de cada unidade experimental foram anestesiados com $75 \mathrm{mg} \mathrm{L}^{-1}$ de benzocaína (Gomes et al., 2001), para a coleta de $1,0 \mathrm{~mL}$ de sangue, por punção caudal, com o auxílio de uma seringa heparinizadadescartável (individual paracadaanimal). Alíquotas de sangue foram destinadas à contagem do número de eritrócitos, hemoglobina e hematócrito, e foram calculados os seguintes índices hematimétricos: hemoglobina corpuscular média (hemoglobina $\times 10 /$ eritrócitos), concentração de hemoglobina corpuscular média (hemoglobina $\times 100 /$ hematócrito) e volume corpuscular (hematócrito $\times 10$ / eritrócitos).

Ao final do período experimental (90 dias), todos os peixes foram capturados, anestesiados, pesados, medidos e contados para o cálculo do desempenho zootécnico quanto a: ganho de massa (massa final massa inicial), ganho de massa diária (ganho de massa/ dias de cultivo), comprimento final, fator de condição de Fulton [massa final/(comprimento final $\left.)^{3} \times 100\right)$ ], conversão alimentar (consumo de ração/ganho de massa), taxa de eficiência alimentar (ganho de massa/ consumo de ração) e sobrevivência [(número de animais no final $\times 100$ ) / número de animais no início].

Oito peixes de cada tanque foram aleatoriamente separados, submetidos à eutanásia com $250 \mathrm{mg} \mathrm{L}^{-1} \mathrm{de}$ benzocaína (Gomes et al., 2001), acondicionados em caixas com gelo e transportados ao laboratório, para avaliação do rendimento corporal do peixe eviscerado [(massa sem vísceras $\times 100) /$ massa total]; relação hepatossomática [(massa do fígado $\times 100) /$ massa total]; e gordura visceral [(massa da gordura das vísceras $\times$ 100) / massa total].

Para as análises de composição química (umidade, proteína bruta, lipídios e matéria mineral), os peixes inteiros foram armazenados em freezer a $-20^{\circ} \mathrm{C}$, conforme Horwitz (2000). Os fígados foram congelados instantaneamente em nitrogênio líquido $\left(-196^{\circ} \mathrm{C}\right)$ e armazenados em freezer a $-20^{\circ} \mathrm{C}$, para posterior análise de deposição de vitamina A e lipídios. Todos os procedimentos foram realizados de acordo com o Comitê de Conduta Ética no Uso de Animais em Experimentação, da Universidade Estadual de Maringá, protocolo $n^{\circ}$ 066/2009.
Os dados foram submetidos ao teste de homogeneidade e normalidade (Cramér-von Mises), e a análise de regressão foi aplicada a $5 \%$ de probabilidade, pelo programa estatístico SAS versão 9.1.3 (SAS Institute, Cary, NC, EUA).

\section{Resultados e Discussão}

Os parâmetros zootécnicos de ganho de massa, ganho de massa diária, comprimento final, fator de condição final, conversão alimentar aparente, eficiência alimentar e sobrevivência não apresentaram diferenças entre as diferentes quantidades de vitamina A na dieta (Tabela 2).

Os resultados quanto ao desempenho zootécnico do pacu se assemelham aos obtidos por Guo et al. (2010), que relataram que a suplementação de vitamina $\mathrm{A}(0,500,1.000,2.000,4.000$ e $8.000 \mathrm{UI})$ não influenciou os parâmetros de desempenho produtivo da tilápia-do-nilo. Thompson et al. (1995) relatam não haver influência da vitamina A sobre o desempenho zootécnico de trutas arco-íris (Oncorhynchus mykiss). Campeche et al. (2009), relatam que o ganho de massa e a conversão alimentar de tilápia-do-nilo apresentou efeito linear diretamente proporcional à quantidade de vitamina A na dieta.

A vitamina A na dieta é essencial para o crescimento e a saúde dos peixes, porém, as respostas nos peixes não seguem um padrão definido. No presente trabalho, não se observou influência da vitamina $\mathrm{A}$ sobre os parâmetros produtivos; no entanto, em trabalho sobre a avaliação de diferentes quantidades de vitamina A na dieta, Guimarães (2009) relatou que tilápias-do-nilo alimentadas com $4.704 \mathrm{UI} \mathrm{kg}^{-1}$ apresentaram o melhor ganho de massa, porém, suplementações superiores a 200 UI kg-1 não influenciaram a conversão alimentar, e a maior retenção proteica foi obtida com $5.300 \mathrm{UI} \mathrm{kg}^{-1}$ de ração. Hu et al. (2006) relataram que o melhor desempenho de tilápia híbrida (Oreochromis niloticus $\times$ O. aureus) foi obtido com 5.850 e $6.970 \mathrm{UI} \mathrm{kg}{ }^{-1}$. Saleh et al. (1995) observaram exigência de 5.000 UI kg-1 em tilápia-do-nilo, o que mostra pouca variação de exigência vitamínica, entre os estudos.

Vários autores ressaltam que, em dieta isenta de vitamina A, é comum ocorrer mortalidade dos peixes (Saleh et al., 1995; Ørnsrud et al., 2002; Hu et al., 2006; Peil et al., 2007; Campeche et al., 2009; Guimarães, 2009). No entanto, não se observou influência sobre a sobrevivência em pacus alimentados

Pesq. agropec. bras., Brasília, v.48, n.8, p.1103-1109, ago. 2013 DOI: $10.1590 / \mathrm{S} 0100-204 \mathrm{X} 2013000800041$ 
com suplementação de vitamina A na dieta, mesmo para aqueles peixes que receberam a ração isenta de suplementação. Guo et al. (2010) também não observaram influência da suplementação de vitamina A sobre a sobrevivência de alevinos de tilápia-do-nilo. Todavia, Guimarães (2009) observou mortalidade de até $77,5 \%$ em tilápias-do-nilo, quando alimentadas com ração isenta de vitamina $\mathrm{A}$ (ração basal composta por farelo de soja, quirera de arroz e celulose), e Peil et al. (2007) observaram até $52,0 \%$ de mortalidade em jundiá (Rhamdia quelen), para os peixes que receberam rações sem suplementação de vitamina A (ração comercial com $56 \%$ de proteína bruta), o que levou à conclusão dos autores de que a mortalidade é a maior expressão da deficiência vitamínica.

Não se observaram diferenças quanto às percentagens de peixe eviscerado, gordura visceral e relação hepatossomática, independentemente da quantidade de vitamina A na ração (Tabela 3). Do mesmo modo, a suplementação de vitamina $\mathrm{A}$ na dieta também não influenciou os parâmetros de composição química da carcaça, em juvenis de pacu cultivados em tanques rede. Vários estudos mostraram particularidades das espécies sobre a composição da carcaça, em peixes alimentados com quantidades crescentes de vitamina A. Os resultados obtidos por Ørnsrud et al. (2002), Mohamed et al. (2003), Hernández et al. (2005) e Hu et al. (2006) ressaltam o aumento da percentagem de proteína na carcaça com a suplementação de vitamina A. Redução da matéria mineral da carcaça, com o aumento da quantidade de vitamina $\mathrm{A}$, foram descritos em estudo sobre juvenis de garoupa Epinephelus tauvina (Mohamed et al., 2003). Por sua vez, o aumento da matéria mineral, em relação à vitamina $\mathrm{A}$, foi descrito em salmão-do-atlântico (Salmo salar L.) (Ørnsrud et al., 2002) e em juvenis de tilápia híbrida (Oreochromis niloticus $\times$ O. aureus) $(\mathrm{Hu}$ et al., 2006). No entanto, em juvenis de falso-alabote-japonês (Paralichthys olivaceus), não foram observadas alterações (Hernández et al., 2005). Estes trabalhos

Tabela 2. Desempenho produtivo dos juvenis de pacu (Piaractus mesopotamicus), alimentados com dieta com diferentes quantidades de vitamina A e cultivados em tanques-rede.

\begin{tabular}{|c|c|c|c|c|c|c|c|}
\hline \multirow[t]{2}{*}{ Parâmetro } & \multicolumn{5}{|c|}{ Vitamina A (UI kg-1 da dieta) } & \multirow{2}{*}{$\begin{array}{l}\mathrm{CV} \\
(\%)\end{array}$} & \multirow[t]{2}{*}{$\mathrm{p}$} \\
\hline & 0 & 3.000 & 6.000 & 9.000 & 12.000 & & \\
\hline Massa inicial (g) & 66,97 & 67,90 & 66,43 & 66,35 & 67,02 & 3,85 & 0,7087 \\
\hline Fator de condição inicial & 2,16 & 2,14 & 2,16 & 2,10 & 2,14 & 3,38 & 0,8364 \\
\hline Ganho de massa (g) & 116,27 & 112,12 & 118,62 & 113,87 & 114,38 & 18,15 & 0,9518 \\
\hline Ganho de massa diária (g) & 1,29 & 1,25 & 1,32 & 1,26 & 1,27 & 18,15 & 0,9518 \\
\hline Comprimento final $(\mathrm{cm})$ & 19,94 & 19,86 & 19,97 & 19,74 & 19,87 & 4,43 & 0,8451 \\
\hline Fator de condição final & 2,30 & 2,28 & 2,31 & 2,33 & 2,31 & 1,83 & 0,9325 \\
\hline Conversão alimentar aparente & 2,52 & 2,76 & 2,52 & 2,57 & 2,59 & 19,46 & 0,9466 \\
\hline Eficiência alimentar & 0,40 & 0,37 & 0,41 & 0,41 & 0,39 & 17,42 & 0,9741 \\
\hline Sobrevivência (\%) & 99,50 & 94,50 & 99,00 & 99,75 & 97,75 & 4,68 & 0,7774 \\
\hline
\end{tabular}

Tabela 3. Rendimento e composição química de carcaça de pacu (Piaractus mesopotamicus) alimentado com dieta com diferentes quantidades de vitamina A e cultivados em tanques rede.

\begin{tabular}{|c|c|c|c|c|c|c|c|}
\hline \multirow[t]{2}{*}{ Parâmetro } & \multicolumn{5}{|c|}{ Vitamina A (UI kg-1 da dieta) } & \multirow{2}{*}{$\begin{array}{l}\mathrm{CV} \\
(\%)\end{array}$} & \multirow[t]{2}{*}{$\mathrm{p}$} \\
\hline & 0 & 3.000 & 6.000 & 9.000 & 12.000 & & \\
\hline \multicolumn{8}{|l|}{ Rendimento (\%) } \\
\hline Eviscerado & 91,00 & 91,54 & 90,71 & 91,40 & 90,01 & 1,22 & 0,3331 \\
\hline Gordura visceral & 3,85 & 4,11 & 3,79 & 4,00 & 4,57 & 9,91 & 0,0696 \\
\hline Relação hepatossomática & 1,11 & 1,10 & 1,22 & 1,16 & 1,22 & 8,25 & 0,2775 \\
\hline \multicolumn{8}{|l|}{ Composição química (\%) } \\
\hline Matéria seca & 31,99 & 33,61 & 31,79 & 33,19 & 32,87 & 4,09 & 0,5704 \\
\hline Umidade & 68,01 & 66,39 & 68,21 & 66,81 & 67,13 & 1,98 & 0,5704 \\
\hline Proteína bruta & 17,60 & 17,58 & 17,84 & 17,31 & 17,19 & 3,09 & 0,2315 \\
\hline Matéria mineral & 9,67 & 11,70 & 9,88 & 10,98 & 11,03 & 14,62 & 0,6806 \\
\hline Gordura & 3,96 & 4,41 & 4,49 & 4,49 & 4,08 & 9,33 & 0,4443 \\
\hline
\end{tabular}


mostram que há uma segregação de resultados, em razão das espécies e das condições experimentais às quais são submetidos os peixes.

A suplementação de vitamina A em ração para pacu não influenciou os parâmetros de gordura dos peixes (Tabela 3). Thompson et al. (1995) e Jeyaraj et al. (2012) também não observaram influência da quantidade de vitamina A sobre a composição da carcaça da truta arco-íris (O. mykiss) e da carpa-comum (Cyprinus carpio). Hu et al. (2006) descreveram que os valores de umidade e proteína da carcaça, em tilápia híbrida (Oreochromis niloticus $\times O$. aureus), reduziram-se com o aumento da vitamina A na dieta, porém, não influenciaram a composição de lipídios e de matéria mineral da carcaça.

No metabolismo de absorção, a vitamina A é absorvida juntamente com as micelas de gordura (Nutrient requirements of warmwater fishes and shellfishes, 1993), contudo, não ocorreu variação no teor de gordura corporal dos peixes. Dedi et al. (1995) também não observaram influência da vitamina $\mathrm{A}$ (10.000 UI kg-1 de ração) sobre o conteúdo hepático no falso-alabote-japonês ( $P$. olivaceus). Estudos que avaliaram a suplementação de vitamina $\mathrm{A}$, em rações para salmão (Ørnsrud et al., 2002), garoupa (Mohamed et al., 2003) e para falso-alabote-japonês (Hernández et al., 2005), observaram redução na gordura da carcaça dos peixes.

As quantidades de vitamina A não influenciaram os resultados hematológicos do pacu (Tabela 4), porém permaneceram dentro dos padrões estabelecidos para a espécie, conforme descrito por Ranzani-Paiva et al. (1999) e Tavares-Dias et al. (2002). Guimarães (2009) relatou que, em dietas isentas de vitamina A, houve redução dos valores de hematócrito, hemoglobina e número de eritrócitos em tilápias- do-nilo. Goswami \& Dutra (1991) relataram que dietas deficientes em vitamina A reduzem os valores de hemoglobina e eritrócitos. Porém, Hernández et al. (2007) e Guo et al. (2010) não observaram diferenças quanto ao hematócrito e à hemoglobina, na avaliação das quantidades de vitamina $\mathrm{A}$ na dieta de juvenis de falso-alabote-japonês e tilápia-do-nilo. Estudos sobre a interação da vitamina A com os parâmetros hematológicos de peixes são incipientes e contraditórios. Em pesquisa sobre a truta arco-íris, Hilton (1983) observou redução da concentração de ferro no fígado dos peixes, com o aumento da quantidade de vitamina A nas dietas, semelhantemente ao que ocorre em mamíferos.

Houve aumento da retenção de vitamina $\mathrm{A}$, nos peixes que receberam rações com 9.000 e 12.000 UI. $\mathrm{O}$ aumento do teor de vitamina no fígado é influenciado pelo conteúdo da ração e pelo período de cultivo (Moren et al., 2004). Os teores de vitamina A das rações foram semelhantes aos relatados na literatura, porém, a não determinação de sinais visuais de deficiência, durante o experimento, se deve ao fato de a ração basal apresentar ingredientes que contêm vitamina $\mathrm{A}$. O tempo de alimentação, para que seja possível detectar os sinais de deficiência, varia conforme a idade dos animais, ou seja, quanto mais jovem o peixe, no início do experimento, mais rapidamente devem surgir os sinais de deficiência, em consequência da quantidade de vitamina A presente no fígado. As tilápias-do-nilo com massa inicial de 5,6 g começaram a apresentar sinais de deficiência em vitamina $A$, com redução da taxa de crescimento aos 21 dias (Guimarães, 2009). Yang et al. (2008) observaram que, após 30 dias de alimentação com a dieta deficiente de vitamina $\mathrm{A}$, as carpas (Cyprinus carpio), com massa inicial de $11,4 \mathrm{~g}$, começaram a apresentar sinais de deficiência.

Tabela 4. Parâmetros sanguíneos de pacu (Piaractus mesopotamicus) alimentados com dieta com diferentes quantidades de vitamina A e cultivados em tanques-rede.

\begin{tabular}{|c|c|c|c|c|c|c|c|}
\hline \multirow[t]{2}{*}{ Parâmetro } & \multicolumn{5}{|c|}{ Vitamina A (UI kg-1 da dieta) } & \multirow{2}{*}{$\begin{array}{l}\text { CV } \\
(\%)\end{array}$} & \multirow[t]{2}{*}{$\mathrm{p}$} \\
\hline & 0 & 3.000 & 6.000 & 9.000 & 12.000 & & \\
\hline Hematócrito (\%) & 36,69 & 37,55 & 37 & 36,34 & 36,84 & 6,13 & 0,8061 \\
\hline Eritrócito $\left(106 \mu \mathrm{l}^{-1}\right)$ & 2,23 & 2,28 & 2,18 & 2,11 & 2,14 & 8,51 & 0,2582 \\
\hline Hemoglobina $\left(\mathrm{g} \mathrm{dL}^{-1}\right)$ & 5,19 & 4,98 & 5,76 & 5,17 & 5,33 & 12,89 & 0,6835 \\
\hline Volume corpuscular médio $\left(\mu^{3}\right)$ & 166,92 & 168,49 & 173,87 & 175,4 & 174,59 & 11,3 & 0,4777 \\
\hline Hemoglobina corpuscular média (pg) & 23,54 & 22,35 & 26,92 & 24,25 & 25,15 & 14,88 & 0,3854 \\
\hline Concentração de hemoglobina corpuscular média (\%) & 14,25 & 13,4 & 15,51 & 14,22 & 14,45 & 13,15 & 0,6667 \\
\hline Vitamina A no fígado (UI kg-1) & 11.745 & 14.255 & 12.693 & 19.690 & 34.557 & - & - \\
\hline Lipídios no fígado $(\%)$ & 5,46 & 5,31 & 5,72 & 5,05 & 6,59 & - & - \\
\hline
\end{tabular}


No presente trabalho, o tamanho dos peixes e o período experimental de 90 dias contribuíram para o não surgimento de sinais clínicos de deficiência de vitamina $\mathrm{A}$, como mortalidade e crescimento reduzido.

\section{Conclusão}

A suplementação de vitamina $\mathrm{A}$, em dietas para juvenis de pacu criados em tanques-rede, não influencia o desempenho produtivo, a composição química, parâmetros sanguíneos e teor de lipídios totais do fígado de pacu (Piaractus mesopotamicus).

\section{Referências}

ABIMORAD, E.G.; CARNEIRO, D.J. Métodos de coleta de fezes e determinação dos coeficientes de digestibilidade da fração protéica e da energia de alimentos para o pacu Piaractus mesopotamicus (Holmberg, 1987). Revista Brasileira de Zootecnia, v.33, p.1101-1109, 2004. DOI: 10.1590/S1516-35982004000500001.

BOYD, C.E. Water quality in ponds for aquaculture. London: Birmingham, 1990. 482p.

CAMPECHE, D.F.B.; CATHARINO, R.R.; GODOY, H.T.; CYRINO, J.E.P.VitaminA in diets for Nile tilapia. ScientiaAgricola, v.66, p.751-756, 2009. DOI: 10.1590/S0103-90162009000600005.

DEDI, J.; TAKEUCHI, T.; SEIKAI, T.; WATANABE, T. Hypervitaminosis and safe levels of vitamin A for larval flounder (Paralichthys olivaceus) fed Artemia nauplii. Aquaculture, v.133, p.135-146, 1995. DOI: 10.1016/0044-8486(95)00015-T.

FERNANDEZ, I.; PIMENTEL, M.S.; ORTIZ-DELGADO, J.B.; HONTORIA, F.; SARASQUETE, C.; ESTÉVEZ, A.; ZAMBONINO-INFANTE, J.L.; GISBERT, E. Effect of dietary vitamin A on Senegalese sole (Solea senegalensis) skeletogenesis and larval quality. Aquaculture, v.295, p.250-265, 2009. DOI: 10.1016/j.aquaculture.2009.06.046.

FURUYA, W.M.; MICHELATO, M.; SILVA, L.C.R.; SANTOS, L.D. dos; SILVA, T.S.C.; SCHAMBER, C.R.; VIDAL, L.V.O.; FURUYA, V.R.B. Fitase em rações para juvenis de pacu (Piaractus mesopotamicus). Boletim do Instituto de Pesca, v.34, p.489-496, 2008.

GOMES, L.C.; CHIPPARI-GOMES, A.R.; LOPES, N.P.; ROUBACH, R.; ARAUJO-LIMA, C.A.R.M. Efficacy of benzocaine as an anesthetic in juvenile tambaqui Colossoma macropomum. Journal of the World Aquaculture Society, v.32, p.426-431, 2001. DOI: 10.1111/j.1749-7345.2001.tb00470.x.

GOSWAMI, U.C.; DUTTA, N.K. Vitamin A-deficient diet and its effects on certain haematological parameters of Heteropneustes fossilis a 3-4-dehydroretinol rich freshwater fish. International Journal for Vitamin and Nutrition Research, v.61, p.205-209, 1991

GUIMARAES, I.G. Vitamina A em dietas para tilápia do Nilo. 2009. 100p. Tese (Doutorado) - Universidade Estadual Paulista, Botucatu.
GUO, R.; LIM, C.; XIA, H.; AKSOR, M.Y.; LI, M. Effect of various dietary vitamin A levels on growth performance and immune response of tilapia (Oreochromis niloticus). Frontiers of Agriculture in China, v.4, p.507-512, 2010. DOI: 10.1007/ s11703-010-1048-0.

HERNÁNDEZ, L.H.H.; TESHIMA, S.-I.; ISHIKAWA, M.; ALAM, S.; KOSHIO, S., TANAKA, Y. Dietary vitamin A requirements of juvenile Japanese flounder Paralichthys olivaceus. Aquaculture Nutrition, v.11, p.3-9, 2005. DOI: 10.1111/j.1365-2 095.2004.00317.x.

HERNÁNDEZ, L.H.H.; TESHIMA， S.-I.; KOSHIO, S.; ISHIKAWA, M.; TANAKA, Y.; ALAM, S. Effects of vitamin A on growth, serum anti-bacterial activity and transaminase activities in the juvenile Japanese flounder, Paralichthys olivaceus. Aquaculture, v.262, p.444-450, 2007. DOI: 10.1016/j. aquaculture.2006.10.012.

HILTON, J.W. Hypervitaminosis A in rainbow trout (Salmo gairdineri): toxicity signs and maximum tolerable level. Journal Nutrition, v.113, p.1737-1745, 1983.

HORWITZ, W. (Ed.). Oficial methods of analysis of AOAC International. $17^{\text {th }}$ ed. Gaithersburg: AOAC International, 2000. $2 \mathrm{v}$.

HU, C.J.; CHEN, S.M.; PAN, C.H.; HUANG, C.H. Effects of dietary vitamin $A$ or $\beta$-carotene concentrations on growth of juvenile hybrid tilapia, Oreochromis niloticus $x$ O. aureus. Aquaculture, v.253, p.602-607, 2006. DOI: 10.1016/j.aquaculture.2005.09.003.

JEYARAJ, N.; RAJAN, M.R.; SANTHANAN, P. Dietary requirement of vitamin $\mathrm{A}$ and biochemical composition of common carp Cyprinus carpio var. cammunis. Journal of Fisheries and Aquatic Science, v.7, p.65-71, 2012. DOI: 10.3923/ jfas.2012.65.71.

JOMORI, R.K.; CARNEIRO, D.J.; MALHEIROS, E.B.; PORTELLA, M.C. Growth and survival of pacu Piaractus mesopotamicus (Holmberg, 1887) juveniles reared in ponds or at different initial larviculture periods indoors. Aquaculture, v.221, p.277-287, 2003. DOI: 10.1016/S0044-8486(03)00069-3.

LALL, S.P.; LEWIS-MCCREA, L.M. Role of nutrients in skeletal metabolism and pathology in fish - an overview. Aquaculture, v.267, p.3-19, 2007. DOI: 10.1016/j.aquaculture.2007.02.053.

LOPERA BARRERO, N.M.; RIBEIRO, R.P.; POVH, J.A.; GOMES, P.C.G.; VARGAS, L.; OLIVEIRA, S.N. de. Caracterización genética de lotes de peces usados en programas de repoblamiento y su importancia en la conservación genética en la piscicultura. Zootecnia Tropical, v.26, p.515-522, 2008.

MOHAMED, J.S.; SIVARAM, V.; CHRISTOPHER ROY, T.S.; MARIAN, M.P.; MURUGADASS, S.; HUSSAIN, M.R. Dietary vitamin A requirement of juvenile greasy grouper (Epinephelus tauvina). Aquaculture, v.219, p.693-701, 2003. DOI: 10.1016/ S0044-8486(02)00665-8.

MOREN, M.; OPSTAD, I.; BERNTSSEN, M.H.G.; ZAMBONINO INFANTE, J.-L.; HAMRE, K. An optimum level of vitamin A supplements for Atlantic halibut (Hippoglossus hippoglossus L.) juveniles. Aquaculture, v.235, p.587-599, 2004. DOI: 10.1016/j. aquaculture.2004.01.030. 
NUTRIENT requirements of warmwater fishes and shellfishes. Washington: National Academic Press, 1993. 114p. (Nutrient requirements of domestic animals).

ØRNSRUD, R.; GRAFF, I.E.; HØIE, S.; TOTLAND, D.K.; HEMRE, G.I. Hypervitaminosis A in first-feeding fry of the Atlantic salmon (Salmo salar L.). Aquaculture Nutrition, v.8, p.7-13, 2002. DOI: 10.1046/j.1365-2095.2002.00185.x.

PEIL, S.Q.; POUEY, J.L.O.F.; LOPES, P.R.S.; MARTINS, C.R.; TIMM, G. Adição de vitamina A na dieta de pós-larvas de jundiá (Rhamdia quelen). Biodiversidade Pampeana, v.5, p.9-15, 2007.

RANZANI-PAIVA, M.J.T.; SALLES, F.A.; EIRAS, J.C.; EIRAS, A.C.; ISHIKAWA, C.M.; ALEXANDRINO, A.C. Análises hematológicas de curimbatá (Prochilodus scrofa), pacu (Piaractus mesopotamicus) e tambaqui (Colossoma macropomum) das estações de piscicultura do Instituto de Pesca, Estado de São Paulo. Boletim do Instituto de Pesca, v.25, p.77-83, 1999.

RODRIGUES, L.A.; FERNANDES, J.B.K.; FABREGAT, T.E.H.P.; SAKOMURA, N.K. Desempenho produtivo, composição corporal e parâmetros fisiológicos de pacu alimentado com níveis crescentes de fibra. Pesquisa Agropecuária Brasileira, v.45, p.897-902, 2010. DOI: 10.1590/S0100-204X2010000800016.
SALEH, G.; ELERAKY, W.; GROPP, J.M. A short note on the effects of vitamin A hypervitaminosis and hypovitaminosis on health and growth of Tilapia nilotica (Oreochromis niloticus). Journal of Applied Ichthyology, v.11, p.382-385, 1995. DOI: 10.1111/j.1439-0426.1995.tb00046.x.

TAVARES-DIAS, M.; MARTINS, M.L.; SCHALCH, S.H.C.; ONAKA, E.M.; QUINTANA, C.I.F.; MORAES, J.R.E. de; MORAES, F.R. de. Alterações hematológicas e histopatológicas em pacu, Piaractus mesopotamicus Holmberg, 1887 (Osteichthyes, Characidae), tratado com sulfato de cobre $\left(\mathrm{CuSO}_{4}\right)$. Acta Scientiarum. Biological Sciences, v.24, p.547-554, 2002.

THOMPSON, I.; CHOUBERT, G.; HOULIHAN, D.F.; SECOMBES, C.J. The effect of dietary vitamin A and astaxanthin on the immunocompetence of rainbow trout. Aquaculture, v.133, p.91-102, 1995. DOI: 10.1016/0044-8486(95)00024-V.

YANG, Q.-H.; ZHOU, X.-Q.; JIANG, J.; LIU, Y. Effect of dietary vitamin A deficiency on growth performance, feed utilization and immune responses of juvenile Jian carp (Cyprinus carpio var. Jian). Aquaculture Research, v.39, p.902-906, 2008. DOI: 10.1111/j.13 65-2109.2008.01945.x.

Recebido em 29 de agosto de 2012 e aprovado em 7 de junho de 2013 